\section{Estilo de vida de adolescentes e sua relação com fatores de risco para Hipertensão Arterial Sistêmica}

\section{Lifestyle of adolescents and their relationship with risk factors for Systemic Arterial Hypertension}

\author{
Eduardo Teixeira Mota Junior, Alexandra Gadelha Medeiros, \\ Raquel Pereira Franklin Thomáz, Ana Vanessa Araújo Pedrosa, \\ Thiago Brasileiro de Vasconcelos, Vasco Pinheiro Diógenes Bastos
}

\section{RESUMO}

O estilo de vida dos adolescentes tem sido associado a comportamentos prejudiciais à saúde que estão diretamente relacionados aos hábitos de vida. Esse estudo objetivou analisar o estilo de vida de adolescentes e a sua relação com os fatores de risco para Hipertensão Arterial Sistêmica, bem como traçar o perfil sociodemográfico; identificar os principais fatores de risco cardiovasculares e investigar os hábitos de vida dos adolescentes relacionados à prevalência da HAS. Tratou-se de um estudo exploratório, descritivo e transversal com estratégia de análise de dados quantitativa, desenvolvido em uma instituição de ensino médio no município de Crateús-CE. Para tanto, foi realizada coleta de dados sociodemográficos, antropométricos e clínicos dos participantes do estudo e aplicado o Questionário Estilo de Vida Fantástico (QEVF). Participaram do estudo 144 estudantes da Escola Estadual de Educação Profissional Manoel Mano, onde $52,77 \%$ ( $n=76$ ) eram do gênero feminino e se auto denominavam de cor parda $(54,86 \%, n=79)$. A idade média dos participantes foi $15,88 \pm 0,08$ anos, sendo que $35,41 \%$ $(n=51)$ possuíam entre 16 e 17 anos. Com relação aos dados clínicos da amostra do estudo foi verificado que os participantes apresentaram valor médio de frequência cardíaca ( $F C)$ de 84,59 $\pm 1,10$ bpm. Considerando a pontuação máxima do QEVF, foi possível evidenciar níveis satisfatórios de qualidade de vida, com média de escore geral de $90 \pm 0,86$ pontos. Face aos resultados obtidos foi possível detectar que os adolescentes participantes do estudo apresentaram em seu estilo de vida fatores de risco para 0 desenvolvimento de HAS: má alimentação e sedentarismo. Todavia também foi verificado que a amostra em estudo apresentou peso corporal e IMC dentro da normalidade, não evidenciando índices para a obesidade. Assim, a proposição de medidas e ações que proporcionem um melhor estilo de vida aos estudantes participantes desse estudo deve ser implantada.

PALAVRAS-CHAVE: Estilo de vida; Adolescente; Hipertensão Arterial Sistêmica.

\section{ABSTRACT}

Adolescents' lifestyles have been associated with health-damaging behaviors that are directly related to lifestyle habits. This study aimed to analyze the lifestyle of adolescents and their relationship with risk factors for Systemic Arterial Hypertension, as well as to outline the sociodemographic profile; identify the main cardiovascular risk factors and investigate the lifestyle habits of adolescents related to the prevalence of SAH. It was an exploratory, descriptive and cross-sectional study with a quantitative data analysis strategy, developed in a high school institution in the municipality of Crateús-CE. For this, sociodemographic, anthropometric and clinical data were collected from the study participants and the Fantastic Lifestyle Questionnaire (QEVF) was applied. 144 students from the Manoel Mano State School of Professional Education participated in the study, where $52.77 \%$ ( $n=76$ ) were female and called themselves brown $(54.86 \%, n=79)$. The average age of the participants was $15.88 \pm 0.08$ years, with $35.41 \%(n=51)$ between 16 and 17 years old. Regarding the clinical data of the study sample, it was found that the participants had an average heart rate $(H R)$ of $84.59 \pm 1.10 \mathrm{bpm}$. Considering the maximum QEVF score, it was possible to evidence satisfactory levels of quality of life, with an average of the general score of $90 \pm 0.86$ points. In view of the results obtained, it was possible to detect that the adolescents participating in the study presented risk factors for the development of SAH in their lifestyle: poor diet and physical inactivity. However, it was also found that the study sample had body weight and $\mathrm{BMI}$ within the normal range, with no evidence of obesity. Thus, the proposal of measures and actions that provide a better lifestyle to the students participating in this study should be implemented. 


\section{INTRODUÇÃO}

A Hipertensão Arterial Sistêmica (HAS) é uma síndrome multifatorial caracterizada por elevados níveis de pressão arterial (PA), associados a alterações metabólicas, hormonais e fenômenos tróficos, como hipertrofia cardíaca e vascular'. A elevação e a manutenção de níveis pressóricos alterados aumentam o risco de doenças cardiovasculares (DCV), das quais a HAS é reconhecida como o principal fator de risco para a morbidade e mortalidade precoce ${ }^{2}$.

A HAS é considerada como um dos maiores problema de saúde pública devido a sua elevada prevalência. 0 crescimento do nú $\urcorner$ mero de pessoas que possuem a HAS tem acarretado custos mé dicos e socioeconômicos elevados, que decorrem principalmente das suas complicações ${ }^{3}$.

Segundo a Organização Mundial da Saúde (OMS), existem cerca de 800 milhões de pessoas com PA elevada em todo o mundo, causando mais de 7 milhões de mortes por ano ${ }^{4}$. No Brasil, inquéritos de base populacional demonstram uma alta prevalência da HAS, com proporções variando de 22,3\% até 43,9\% da população adulta. Estas altas prevalências encontradas em adultos são preocupantes, contudo, a literatura nacional tem observado prevalências de HAS em crianças e adolescentes variando de $3,6 \%$ a $21,2 \%{ }^{5}$.

A HAS é comumente classificada em dois tipos: primária, com causa desconhecida, e secundária, com causa orgânica claramente desencadeadora da elevação dos valores pressóricos ${ }^{6,7}$. Entre os diversos indicadores, esta doença está associada a diversos fatores de risco ambientais como os baixos níveis de atividade física, o tabagismo, o consumo excessivo de sal e de álcool, além do histórico familiar, a diabetes e o nível socioeconômico, tanto em adultos quanto em adolescentes ${ }^{5}$.

Estudos longitudinais têm demonstrado que crianças com níveis de PA elevados apresentam maior probabilidade de se tornarem adultos portadores de HAS. Um estudo mostrou que os valores iniciais altos durante a infância estiveram correlacionados positivamente com os valores da PA sistólica e diastólica quatro anos mais tarde ${ }^{8}$.

O estilo de vida de crianças e adolescentes tem sido associado a comportamentos prejudiciais à saúde, como experimentação de bebidas alcóolicas e tabagismo. Adicionalmente, o hábito de assistir televisão pode influenciar as escolhas alimentares dos adolescentes, uma vez que a maioria dos alimentos veiculados pela mídia é de alta densidade energética, contribuindo para o aumento da obesidade e HAS entre eles ${ }^{9}$.

Ações de promoção da saúde relacionadas a mudanças de estilo de vida representam a possibilidade de prevenção mais efetiva da ocorrência de eventos cardiovasculares. Os estudos relativos à detecção dos indicadores de risco em populações jovens são essenciais para o acompanhamento dos indivíduos que apresentam maior risco de alterações na idade adulta ${ }^{10,11}$.

A prevenção da HAS se dá através de algumas medidas aplicadas nas diversas etapas da evolução da história do agravo à saúde. A prevenção primária tem início antes da doença se manifestar, através de práticas de educação em 
saúde. A prevenção secundária se aplica no decorrer da doença, com tratamento medicamentoso e mudanças de hábitos de vida. E por último se destaca a prevenção terciária, que está relacionada à reabilitação de pacientes com sequelas ou limitações físicas originadas de complicações cardiovasculares ${ }^{12,13}$.

Compreendendo que a ocorrência de elevados níveis de PA na adolescência, atribuem-se ao fato de que quanto mais precocemente os desfechos hipertensivos forem detectados maior será a possibilidade de uma intervenção eficaz, julgamos oportuno averiguar o estilo de vida de adolescentes em relação ao risco de HAS.

Dessa forma, essa pesquisa torna-se relevante devido ao fato deste estudo contribuir para a produção de novos conhecimentos sobre os aspectos preventivos em adolescentes em relação à HAS e também para que possa desenvolver uma maior assistência a essa população. Portanto o estudo objetivou analisar o estilo de vida de adolescentes e a sua relação com os fatores de risco para HAS, bem como traçar o perfil sociodemográfico; identificar os principais fatores de risco cardiovasculares e investigar os hábitos de vida dos adolescentes relacionados a prevalência da Hipertensão.

\section{MÉTODO}

Tratou-se de um estudo exploratório, descritivo e transversal com estratégia de análise de dados de forma quantitativa. O estudo foi realizado na Escola Estadual de Educação Profissional Manoel Mano, Crateús - CE, 63700000. A coleta de dados foi realizada no mês de outubro de 2016, mediante a aprovação do Comitê de Ética em Pesquisa do Centro Universitário Estácio do Ceará (Protocolo n 1.784.946).

A população foi composta por estudantes da referida Escola, na qual tem matriculado 538 alunos, ficando amostra em 144 estudantes, baseado em cálculo para amostra finita. Foi considerado como critério de inclusão estar regulamente matriculado na instituição de ensino durante o ano letivo de 2016, ter idade de 12 a 18 anos independente do gênero, raça e condição civil, desde que aceitem fazer parte da pesquisa, sendo excluídos do estudo os estudantes que se oponham a participação do estudo e os que faltarem no dia da apresentação do projeto.

Foi feita uma visita à Escola Estadual de Educação Profissional Manoel Mano, na qual foram expostos os objetivos da pesquisa, visando à autorização da mesma. Após aprovação do Comitê de Ética e Pesquisa, a coleta de dados foi realizada mediante a abordagem dos alunos da Escola em participante da pesquisa, onde foi explicado o procedimento do estudo aos estudantes e assinado o Termo de Assentimento Livre e Esclarecido e solicitado aos estudantes que levassem o Termo de Consentimento Livre e Esclarecido para os Pais/Responsáveis para ser assinado.

Em outra oportunidade, que corresponde ao dia do recebimento dos Termos (Assentimento e Consentimento) foi realizada a aplicação do Questionário Estilo de Vida Fantástico (QEVF) que se trata de um instrumento genérico que foi desenvolvido no Departamento de Medicina Familiar da Universidade McMaster, no Canadá, com a finalidade de auxiliar 
os médicos que trabalham com a prevenção, a fim de que estes pudessem conhecer e medir o estilo de vida de seus pacientes. Esse questionário possui 25 perguntas relacionadas ao estilo de vida ${ }^{14}$.

A soma de todos os pontos resultantes de todos os domínios permite chegar a um score global que classifica os indivíduos de 0 a 125 pontos. Sendo que cada domínio do QEVF é constituído por questionamentos pontuados que podem ser pontuados de 0 a 5 pontos. Esse questionário teve intitulação originária da palavra "fantástico" vem do acrônimo "fantastic", que representa as letras dos nomes dos nove domínios, enumerados a seguir 15,16 .

$$
\begin{aligned}
& \text { F = "family and friends" (escore: mínimo 02, máximo } 10 \text { pontos); } \\
& \text { A = "activity" (escore: mínimo 02, máximo } 10 \text { pontos); } \\
& \text { N = "nutrition" (escore: mínimo 03, máximo } 15 \text { pontos); } \\
& \text { T = "tobacco and toxics" (escore: mínimo 04, máximo } 20 \text { pontos); } \\
& \text { A = "alcohol" (escore: mínimo 03, máximo } 15 \text { pontos); } \\
& \text { S = "sleep", "seat belts", "stress", "sex" (escore: mínimo 05, máximo } 25 \text { pontos); } \\
& \text { T = "type of behavior" (escore: mínimo } 02, \text { máximo } 10 \text { pontos); } \\
& \text { I = "insight" (escore: mínimo 03, máximo } 15 \text { pontos); } \\
& \text { C = "career" (escore: mínimo 01, máximo } 05 \text { pontos). }
\end{aligned}
$$

Além da avaliação do estilo de vida realizada pela aplicação do QEVF, também foi realizada a coleta de dados sociodemográficos (idade, gênero, escolaridade e raça), antropométricos (altura e peso) e clínicos dos participantes (presença de comorbidades e/ou patologias associadas).

O Guia para universidades saludables y otras instituciones de educación superior ${ }^{17}$ propõe cinco níveis de classificação que estratificam o comportamento em: de 0 a 46 (Necessita melhorar); de 47 a 72 (Regular); de 73 a 84 (Bom); de 85 a 102 (Muito bom) e de 103 a 125 (Excelente).

Os dados foram analisados a partir da estatística descritiva e inferencial através do software estatístico programa SPSS® versão 13.0 (SPSS® Inc; Ilinois, USA).

\section{RESULTADO}

Participaram do estudo 144 estudantes da Escola Estadual de Educação Profissional Manoel Mano, onde 52,77\% ( $n=76$ ) eram do gênero feminino e se auto denominavam de cor parda $54,86 \%$ ( $n=79)$. A idade média dos participantes foi $15,88 \pm 0,08$ anos, sendo que 35,41\% ( $n=51$ ) possuíam entre 16 e 17 anos (Tabela 1). 
Tabela 1. Distribuição dos dados de acordo com o perfil sócio demográfico dos participantes do estudo ( $n=144)$. FortalezaCE/2016.

\begin{tabular}{llll}
\hline \multicolumn{1}{l}{ Variáveis } & $\mathbf{N}$ & $\%$ \\
\hline \multicolumn{1}{c}{ Gênero } & & \\
& Feminino & 76 & $52,77 \%$ \\
& Masculino & 68 & $47,23 \%$ \\
& TOTAL & 144 & $100,00 \%$ \\
\hline Idade & & & \\
& $14-15$ & 8 & $5,55 \%$ \\
& $15-16$ & 47 & $32,63 \%$ \\
& $16-17$ & 51 & $35,41 \%$ \\
& $17-18$ & 30 & $20,83 \%$ \\
& $18-19$ & 8 & $5,55 \%$ \\
& TOTAL & 144 & $100 \%$ \\
\hline Raça & Branca & & \\
& Preta & 46 & $31,95 \%$ \\
& Amarela & 7 & $4,86 \%$ \\
Parda & 7 & $4,86 \%$ \\
Indígena & 79 & $54,86 \%$ \\
TOTAL & 5 & $3,47 \%$ \\
\hline
\end{tabular}

Com relação aos dados clínicos da amostra do estudo foi verificado que os participantes apresentaram valor médio de frequência cardíaca (FC) de 84,59 $\pm 1,10$ bpm, PA sistólica de 122,55 $\pm 1,45 \mathrm{cmH}_{2} \mathrm{O}$ e diastólica de 78,45 $\pm 1,09 \mathrm{cmH}_{2} \mathrm{O}$.

A média do peso corporal dos participantes era de 59,53 $\pm 0,95 \mathrm{Kg}$, com IMC médio de 21,47 $\pm 0,31$. Na amostra foi evidenciado que 68,1\% ( $n=98)$ dos estudantes estavam com o peso normal e 2\% ( $n=3$ ) apresentavam obesidade (Gráfico 1). Gráfico 1. Distribuição dos dados de acordo com o índice de massa corpórea (IMC) dos participantes do estudo (n=144).

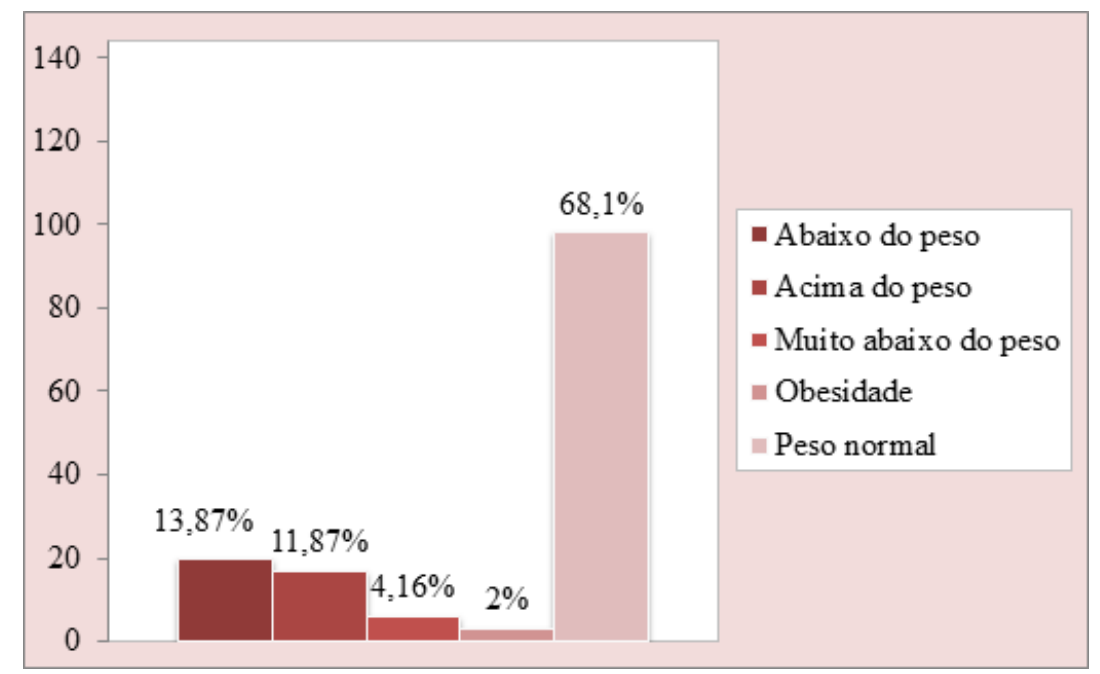


Considerando que a pontuação máxima do QEVF é 125 pontos e que quanto menor o escore pior o estilo de vida, foi possível evidenciar que foram analisados níveis satisfatórios durante a análise, onde na qual a média de escore geral de todos os participantes foi de $90 \pm 0,86$ pontos.

Com relação às dimensões avaliadas pelo QEVF, foi verificado que na dimensão de Atividade Física, os estudantes apresentaram em média uma pontuação baixa (4,83 \pm 0,20 ponto), sendo que a pontuação máxima da dimensão era 10 pontos. Outro domínio que apresentou resultados baixos foi a Nutrição com pontuação média de 9,69 \pm 0,21 pontos, na qual a pontuação máxima esperada seria de 15 pontos (Tabela 2).

Quando analisados os domínios com relação ao gênero, foi evidenciada pontuação do domínio sono, estresse e sexo, que as mulheres apresentaram pontuação média de 16,46 \pm 0,34 pontos. Já os homens, obtiveram valor médio de 3,75 \pm 0,12 pontos no domínio Trabalho, sendo que as mulheres apresentaram pontuação média também inferior, de $3,51 \pm 0,14$ pontos (Tabela 2).

Tabela 2. Distribuição dos dados de acordo com as dimensões do estilo de vida dos estudantes $(n=144)$. FortalezaCE/2016.

\begin{tabular}{lllll}
\hline & Dimensão & Masculino $(\mathbf{n}=68)$ & Feminino $(\mathbf{n}=\mathbf{7 6})$ & Total $(\mathbf{n}=\mathbf{1 4 4})$ \\
\hline $\mathbf{F}$ & Família e amigos & $8,10 \pm 0,13$ & $7,88 \pm 0,17$ & $7,99 \pm 0,16$ \\
$\mathbf{A}$ & Atividade física & $5,53 \pm 0,21$ & $4,21 \pm 0,17$ & $4,83 \pm 0,20$ \\
$\mathbf{N}$ & Nutrição & $9,76 \pm 0,22$ & $9,62 \pm 0,21$ & $9,69 \pm 0,21$ \\
T & Tabaco e drogas & $18,51 \pm 0,12$ & $19,00 \pm 0,09$ & $18,77 \pm 0,11$ \\
$\mathbf{A}$ & Álcool & $13,51 \pm 0,19$ & $14,20 \pm 0,12$ & $13,88 \pm 0,16$ \\
$\mathbf{S}$ & Sono, estresse e sexo & $17,65 \pm 0,33$ & $16,46 \pm 0,34$ & $17,02 \pm 0,34$ \\
T & Trabalho & $3,75 \pm 0,12$ & $3,51 \pm 0,14$ & $3,63 \pm 0,13$ \\
I & Introspeção & $11,04 \pm 0,21$ & $10,71 \pm 0,21$ & $10,87 \pm 0,21$ \\
C & Comportamento & $7,00 \pm 0,14$ & $6,99 \pm 0,16$ & $6,99 \pm 0,15$ \\
\hline
\end{tabular}

De acordo com os níveis de classificação de estratificação de comportamento, $61,11 \%(n=88)$ dos participantes do estudo obtiveram classificação considerada como "muito boa", sendo que apenas 4,87\% apresentaram classificação "regular" e nenhum dos participantes obtiveram resultados classificados com "necessita melhorar". No entanto quando analisados os resultados com relação ao genero dos participantes verificou-se que $6,58 \%(n=5)$ das mulheres apresentaram classificação do estilo de vida com regular (Gráfico 2). 
Gráfico 2. Distribuição da classificação do estilo de vida dos participantes do estudo de acordo com o escore obtido pelo Questionário de Estilo de Vida Fantástico ( $n=144)$.

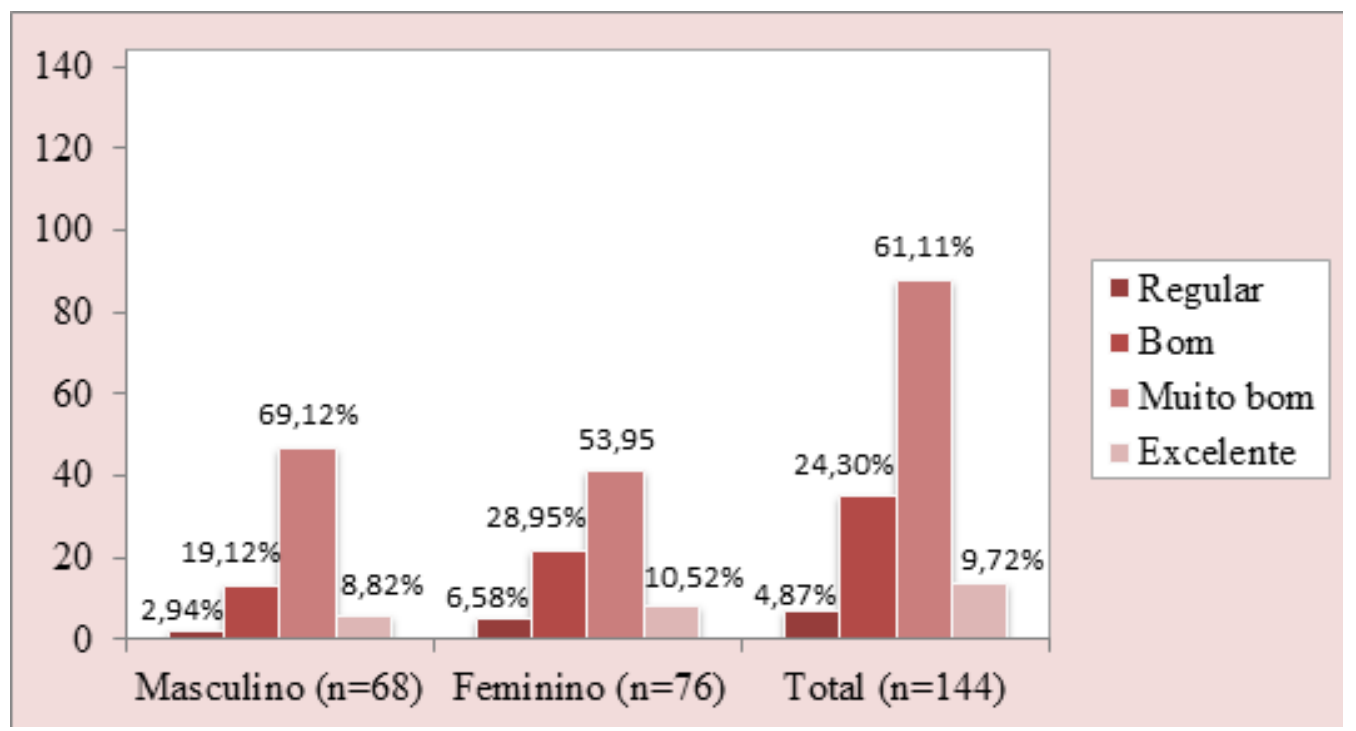

\section{DISCUSSÃO}

Os resultados obtidos nesta pesquisa identificaram uma prevalência de adolescentes do gênero feminino na Escola Estadual de Educação Profissional Manoel Mano, achados que vão de encontro com os dados do estudo realizado por Hackenhaar et al.18 que verificou uma proporcionalidade dos gêneros feminino e masculino de 2:1, assinalando uma predominância do gênero feminino em nível estudantil.

Também foi identificado na amostra em estudo preponderância de adolescentes com idade entre 16 e 17 anos e que possuíam raça parda. Dados semelhantes aos achados de Dias et al. ${ }^{19}$ que identificou em sua amostra predomínio de estudantes adolescentes com faixa etária de 16 a 17 anos.

No que diz respeito aos dados clínicos da amostra do estudo foi evidenciado níveis de PA sistólica e diastólica dentro dos valores considerados como normais, dados que diferem dos achados de Kroth e Maia ${ }^{20}$ que verificaram prevalência de níveis pressóricos acima da normalidade em sua amostra. Em outro estudo desenvolvido por Chaves et al. ${ }^{21}$ com 141 adolescentes em Fortaleza/CE, também evidenciou elevados níveis pressóricos, sendo que as medidas de PA foram maiores nos indivíduos do gênero masculino que no feminino.

Quanto ao IMC dos participantes do estudo, foi verificado que 68,1\% ( $\mathrm{n}=98)$ dos estudantes estavam com o peso normal. Dados que vão de encontro aos achados de Nobre et al. ${ }^{22}$, que ao avaliar a relação do IMC e da circunferência de cintura (CC) sobre a PA de adolescentes, observou que o percentual de participantes com PA elevada foi significativamente mais alto entre aqueles com maior Circunferência da Cintura.

Sendo que Hoehr et al. ${ }^{23}$ verificam em seu estudo correlação positiva entre níveis elevados de PA e obesidade em adolescentes com CC elevada. Dados semelhantes aos achados de Freitas et al. ${ }^{24}$ que define as medidas de CC 
como um indicador fortemente relacionado ao desenvolvimento de HAS é a obesidade, que constitui um problema de saúde crescente na população infanto juvenil em várias partes do mundo.

Já Costa et al. ${ }^{25}$ afirmam que são vários os fatores envolvidos no desenvolvimento da HAS durante toda a vida, dentre eles a obesidade, o sedentarismo e os hábitos alimentares inadequados, que estão no topo dos principais fatores de risco cardiovasculares (FRCV) que sensibilizam o organismo a desenvolver essa doença.

Todavia, Al-Hazzaa et al. ${ }^{26} \mathrm{e}$ Craemer et $a l .{ }^{27}$ afirmam que a relação das atividades sedentárias com a prática de atividade física é independente, visto que um indivíduo pode praticar as atividades sedentárias e seguir as recomendações de atividade física simultaneamente. Desse modo a prática de atividade física exerceu efeito independente sobre os comportamentos sedentários dos adolescentes participantes do estudo.

Quanto aos hábitos alimentares, os participantes do estudo apresentaram resultados baixos quanto ao aspecto nutricional. No entanto, Costa et al. ${ }^{25}$ relata que os adolescentes estão diretamente expostos aos FRCV, principalmente no que concerne a problemas nutricionais, como o excesso de peso e o desenvolvimento de obesidade precoce que precedem a HAS.

Entretanto, Freitas et al. ${ }^{24}$ menciona a ingestão de álcool, por períodos prolongados de tempo, como um forte FRCV relacionado à prevalência de DCV em jovens. Segundo Sociedade Brasileira de Cardiologia', a ingestão de bebidas alcóolicas podem aumentar a PA e a mortalidade cardiovascular em geral. Todavia o consumo de álcool e outras drogas não foi evidenciado nesse estudo com FRCV relevante.

Freitas et al. ${ }^{24}$ também afirma que os FRCV constituem determinantes indispensáveis do estilo de vida. Foi verificado nesse estudo que dentre os participantes, $61,11 \%(n=88)$ evidenciaram níveis de estilo de vida considerados "muito bom", dados semelhantes ao estudo de Leite e Santos ${ }^{15}$ que também avaliou o estilo de vida de 244 estudantes no sul do país por meio do QEVF.

\section{CONSIDERAÇÕES FINAIS}

Face aos resultados obtidos foi possível observar que os adolescentes participantes do estudo apresentaram em seu estilo de vida fatores de risco para o desenvolvimento de Hipertensão Arterial Sistêmica, má alimentação e sedentarismo. Todavia também foi verificado que a amostra em estudo apresentou peso corporal e IMC dentro da normalidade, não evidenciando índices para a obesidade.

Nessa perspectiva, a proposição de medidas e ações que proporcionem um melhor estilo de vida aos adolescentes participantes desse estudo deve ser implantada como alternativa para a garantia da saúde individual e coletiva desses estudantes. 


\section{REFERÊNCIAS}

1. Sociedade Brasileira de Cardiologia. Sociedade Brasileira de Hipertensão. Sociedade Brasileira de $\mathrm{Ne}$ frologia. VI Diretrizes Brasileiras de Hipertensão. Arq Bras Cardiol 2010; 95(1): 1-51.

2. Ferreira SD, Carballo FP, Sousa FF, Silva DMR. Prevalência e fatores associados ao sobrepeso/obesidade e à hipertensão arterial sistêmica em crianças da rede privada de ensino de Divinópolis/MG. Cad. Saúde Colet. 2015; 23(3): 289-297.

3. Lima AS, Araújo RC, Gomes MRA, Schwingel PA, Pitangui ACR. Prevalência de hipertensão e sua associação com excesso de peso e atividade física em adolescentes. ABCS Health Sci. 2014; 39(2): 83-87.

4. Moreira NF, Muraro AP, Brito FSB, Silva RMVG, Rosely Sichieri R, Ferreira MG. Obesidade: principal fator de risco para hipertensão arterial sistêmica em adolescentes brasileiros participantes de um estudo de coorte. Arq Bras Endocrinol Metab. 2013; 57(7): 520-526.

5. Bozza R, Barbosa Filho VC, Maziero RSB, Bontorin MS, Campos W. Relação entre somatório de dobras cutâneas e pressão arterial sistêmica em adolescentes. Rev Bras Promoç Saúde. 2014; 27(2): 263-268.

6. Ferreira JS, Aydos RD. Prevalência de hipertensão arterial em crianças e adolescentes obesos. Ciência \& Saúde Coletiva. 2010; 15(1): 97-104.

7. Corrêa Neto VG, Sperandei S, Silva LAI, Maranhão Neto GA, Palma A. Hipertensão arterial em adolescentes do Rio de Janeiro: prevalência e associação com atividade física e obesidade. Ciência \& Saúde Coletiva. 2014; 19(6): 1699-1708.

8. Vasconcelos TB, Lima AT, Farias MSQ, Câmara TMS, Pontes SMM, Souza CT et al. Estilo de vida de adolescentes das escolas públicas de ensino fundamental, em Fortaleza/CE, em relação ao risco de hipertensão. J Health Biol Sci. 2013; 1(1): 10-15.

9. Dias PJP, Domingos IP, Ferreira MG, Muraro AP, Sichieri R, Silva RMVG. Prevalência e fatores associados aos comportamentos sedentários em adolescentes. Rev Saúde Pública. 2014; 48(2): 266-274. 
10. Araújo TL, Lopes MVO, Cavalcante TF, Guedes NG, Moreira RF, Chaves EM, et al. Análise de indicadores de risco para hipertensão arterial em crianças e adolescentes Rev Esc Enferm USP. 2008; 42(1): 120-6.

11. Wenze D, Souza JMP, Souza SB. Prevalência de hipertensão arterial em militares jovens e fatores associados. Rev Saúde Pública. 2009; 43(5): 789-95.

12. Silva LR, Oliveira EAR, Lima LHO, Formiga LMF, Sousa ASJ, Silva RN. Fatores de risco para hipertensão arterial em policiais militares do centro-sul piauiense. Revista Baiana de Saúde Pública. 2014; 38(3): 679-692.

13. Mendes GS, Moraes CF, Gomes L. Prevalência de hipertensão arterial sistêmica em idosos no Brasil entre 2006 e 2010. Rev Bras Med Fam Comunidade. 2014; 9(32): 273-278.

14. Wilson DMC, Nielsen E, Ciliska D. Lifestyle assessment: testing the Fantastic Instrument. Canadian Family Physician,1984; 30: 1.863-1.866.

15. Leite TRA, Santos BRM. Pressão arterial e estilo de vida de estudantes universitários. Revista Brasileira de Ciências da Saúde, 2011; 9(27):14-20.

16. Silva AMM, Brito IS, Amado JMC. Tradução, adaptação e validação do questionário Fantastic Lifestyle Assessment em estudantes do ensino superior. Ciência \& Saúde Coletiva, 2014; 19(6):1901-9.

17. Lange I, Vio F. Guía para universidades saludables y otras instituciones de educación superior. Santiago de Chile; INTA/Universidad de Chile; 2006.

18. Hackenhaarl ML, Sichierill R, Muraro AP, Silva RMVG, Ferreira MG. Mobilidade social, estilo de vida e índice de massa corporal de adolescentes. Rev Saúde Pública. 2013; 47(5):942-51.

19. Dias PJP, Domingos IP, Ferreira MG, Muraro AP, Sichieri R, Silva RMVG. Prevalência e fatores associados aos comportamentos sedentários em adolescentes. Rev Saúde Pública. 2014; 48(2):266-274.

20. Kroth JB, Maia HMSF. Pressão arterial, perfil antropométrico e demais fatores de risco cardiovascular em escolares da rede pública. Revista de Pesquisa em Fisioterapia. 2015; 5(3):251-261. 
21. Chaves ES, Araújo TL, Chaves DBR, Costa AGS, Oliveira ARS, Alves FEC. Crianças e adolescentes com história familiar de hipertensão arterial: indicadores de risco cardiovasculares. Acta Paul Enferm. 2009; 22(6):793-9.

22. Nobre NL, Sammour SNF, Sobrinho PSC. Índice de massa corporal e circunferência de cintura como preditores de pressão arterial alterada em adolescentes. Rev Med Minas Gerais. 2011; 21(4):404-12.

23. Hoehr CF, Reuter CP, Tornquist L, Nunes HMB, Burgos MS. Prevalência de obesidade e hipertensão arterial em escolares: estudo comparativo entre escolas rurais do município de Santa Cruz do Sul/RS. Revista de Epidemiologia e Controle de Infecção.2014; 4(2): 122-126.

24. Freitas D, Rodrigues CS, Yagui CM, Carvalho RST, Alves LMM. Fatores de risco para hipertensão arterial entre estudantes do ensino médio. Acta Paul Enferm. 2012; 25(3): 430-4.

25. Costa JV, Silva ARV, Moura IH, Carvalho RMN, Bernardes LE, Almeida PC. Análise de fatores de risco para hipertensão arterial em adolescentes escolares. Rev. Latino-Am. Enfermagem. 2012; 20(2): 1-7.

26. Al-Hazzaa HM, Abahussain NA, Al-Sobayel HI, Qahwaji DM, Musaiger AO. Physical activity, sedentary behaviors and dietary habits among Saudi adolescents relative to age, gender and region. Int J Behav Nutr Phys Act. $2011 ; 8: 140$.

27. Craemer M, Decker E, Bourdeaudhuiji I, Vereecken C, Deforche B, Manios Y, et al. Correlates of energy balance-related behaviours in preschool children: a systematic review. Obes Rev. 2012; 13(Suppl 1):13-28. 